\title{
Effect of ENSO on Queensland seasonal landfalling tropical cyclone activity
}

\author{
Shaun Flay* and Jonathan Nott \\ School of Tropical Environment Studies and Geography, James Cook University, Australia, \\ School of Earth and Environmental Sciences, James Cook University, Australia
}

\begin{abstract}
:
A statistical model for predicting seasonal tropical cyclone landfalls in Queensland, Australia using an index of the El Niño-Southern Oscillation (ENSO) is presented. The approach uses a generalised linear model (GLM) to relate seasonal counts to the Southern Oscillation Index (SOI). A Bayesian methodology is employed to estimate parameters of the model. The available tropical cyclone record is first separated into historical (1910/1911-1959/1960) and instrumental (1960/1961-2004/2005) eras. Historical counts, which are considered to be less reliable observational sources, are used to specify informative prior distributions when fitting the GLM to instrumental counts with the Bayesian approach. The inclusion of historical information is found to lead to increased certainty in parameter estimates when compared to a model where historical counts are excluded and a non-informative prior model used. Predictive distributions are given, which allow inferences on seasonal landfall activity, given pre-seasonal values of the SOI. A cross-validation procedure shows that the model incorporating historical information outperforms, in terms of mean-squared prediction error, both the non-informative prior model and a model without the SOI-predictor (climatology). A trend analysis highlights possible decadal variability in the relationship between ENSO and seasonal tropical cyclone activity. Copyright (c) 2007 Royal Meteorological Society
\end{abstract}

KEY WORDS landfalling tropical cyclones; Queensland; ENSO; generalised linear model; Bayesian; seasonal prediction

Received 4 July 2006; Revised 19 September 2006; Accepted 24 September 2006

\section{INTRODUCTION}

Australian region tropical cyclone activity exhibits marked inter-seasonal variability. Nicholls (1979) first showed that part of this variability was explained by pressure anomalies at Darwin, an index of the El NiñoSouthern Oscillation (ENSO) phenomena, in the months preceding the tropical cyclone season. Nicholls (1984) subsequently demonstrated a link to ENSO indices based on sea surface temperatures (SSTs), finding that tropical cyclone numbers were generally greater in seasons preceded by high SSTs in the north Australian region and low SSTs in the east equatorial Pacific. Since then, several attempts have been made to produce seasonal forecast models for Australian region tropical cyclone activity using a lead value of the Southern Oscillation Index (SOI) (e.g. Nicholls, 1992; Nicholls et al., 1998; McDonnell and Holbrook, 2004).

Considerable effort has also been directed towards investigating the relationship between ENSO and tropical cyclone activity in the Coral Sea (Figure 1) and broader southwest Pacific regions (e.g. Revell and Goulter, 1986; Basher and Zheng, 1995; Grant and Walsh, 2001). Basher and Zheng (1995) showed that the reduced

* Correspondence to: Shaun Flay, Willis Reinsurance, Level 4, 1 Castlereagh Street, Sydney, Australia, 2000. E-mail: flays@willis.com (increased) incidence of storm activity in the Coral Sea region during El Niño (La Niña) periods is largely associated with localised SST variability. During El Niño periods, positive SST anomalies tend to occur further eastward towards the central Pacific causing an eastward displacement in the origins of tropical cyclones. This results in an effective decrease in tropical cyclone activity nearer Queensland and consequently fewer landfall events (Grant and Walsh, 2001). During La Niña periods, the reverse pattern occurs and there is a corresponding increase in activity near Queensland and a higher number of landfall events.

Relatively little work has been undertaken to statistically model the relationship between Queensland landfalling tropical cyclones and ENSO. This is somewhat surprising because this landfall record can be considered fairly reliable for the period prior to the advent of routine satellite reconnaissance in the 1960s. As a consequence of concerns over the Australian region best-track database detailed by Holland (1981), most recent studies for the region have restricted their analyses to the post1960 period or later (e.g. Broadbridge and Hanstrum, 1998; Nicholls et al., 1998; McDonnell and Holbrook, 2004). Given that this represents a relatively short time period, any statistical analysis undertaken on this record is potentially subject to considerable uncertainties. 
Several strategies have been proposed to incorporate sources of historical information when analysing seasonal tropical cyclone activity. In fitting a Poisson regression model to storm counts in the Australian region with the SOI as a predictor, Solow and Nicholls (1990) treated counts prior to 1965 as incomplete. They then proposed a model for estimating the likelihood that an event was observed during the pre-1965 period as a means to reconstruct the incomplete portion of the record. More recently, Elsner and Bossak (2001) highlighted the advantages of Bayesian statistical methods as ideally suited for combining instrumental hurricane records with historical records in the United States. Elsner and Jagger (2004) extended this Bayesian modelling strategy to the prediction of seasonal US hurricane activity conditional on several climate indices.

This study looks specifically at tropical cyclones making landfall along the Queensland coast rather than the entire Australian or southwest Pacific/Coral Sea regions. In terms of methodology, the major difference with previous studies conducted in the Australian region is in the adoption of a Bayesian modelling approach. The advantages of this approach for this study are twofold. First, as shown by Elsner and Bossak (2001) and Elsner and Jagger (2004), it provides a means to combine sources of historical observations with information from a period of sampling that is considered reliable. The use of an extended record thus offers an opportunity to quantify any relationship between seasonal landfall activity and ENSO with greater confidence. Second, the availability of a posterior distribution for model parameters can more readily facilitate predictive inference on future storm activity within a probabilistic framework. A cross-validation procedure is also employed here to compare predictions derived from the model that includes historical counts against a model that uses only counts from the instrumental record. These models are also compared against predictions from a model based on climatology without the ENSO predictor. In addition, the possibility of trends in the relationship between ENSO and tropical cyclone landfalls over the twentieth century is examined.

\section{DATA}

In the Australian region, a tropical cyclone is defined as a non-frontal, synoptic-scale system that has developed over tropical waters and has a 10-min mean surface wind speed of at least $63 \mathrm{~km} / \mathrm{h}$ near the centre of an organised wind circulation. The best-track database archived by the Bureau of Meteorology (BoM) compiles observations of these events in the Australian region $\left(\sim 90^{\circ} \mathrm{E}-160^{\circ} \mathrm{E}\right)$. This data set is available in an electronic format at http://www.bom.gov.au/climate/how/. To ensure maximum accuracy, observations within this database were cross-referenced with information from the Queensland region $\left(\sim 135^{\circ} \mathrm{E}-165^{\circ} \mathrm{E}\right)$ tropical cyclone database, which was obtained from the Severe Weather Section of the BoM in Brisbane. In addition to this, a summary record of Queensland tropical cyclone impacts (Callaghan, 2005) was used to verify the occurrence of landfall events, particularly in the case of those occurring prior to the 1960s.

A landfall was defined here as a tropical cyclone originating in the Coral Sea and crossing the mainland east coast (Figure 1). This definition also included major near-coast islands. Note also that multiple landfalls of the same tropical cyclone were counted as a single event. A distinction was made between an instrumental era (1960/1961-2004/2005) and a historical era (1910/1911-1959/1960) in the best-track observations. The former represents the beginning of a formal structure in the tracking and recording of tropical cyclones, whereas the latter represents a period in which sampling

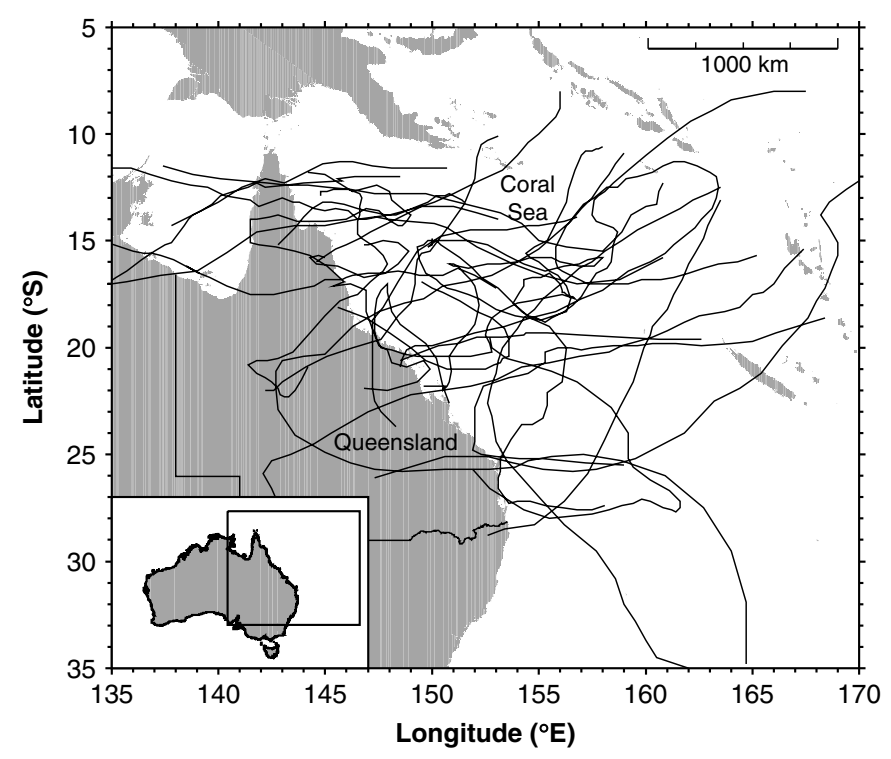

Figure 1. Coral Sea tropical cyclones making landfall along the east coast of Queensland over the period 1960/1961-2004/2005. Note that this figure includes only events that attained a minimum central pressure of at least $990 \mathrm{hPa}$ at some stage during their lifetime. 
was largely ad hoc in nature. The period post-1960 has been used in a number of recent studies (e.g. Broadbridge and Hanstrum, 1998; McDonnell and Holbrook, 2004) as the date for which reliable data on tropical cyclones in the Australian region are available.

As a consequence of major improvements in the observational network in the second half of the last century, tropical cyclone observations in the Australian region are generally acknowledged to be sufficiently reliable only from the 1960s onwards (Holland, 1981). Before this, the detection and monitoring of tropical cyclones relied on limited surface measurements from ships and land stations. During this period, a number of events, particularly those that remained well out at sea for their duration, were liable to go undetected. Conversely, it is also likely that some low-pressure weather systems were mis-classified as tropical cyclones due in part to inadequate information.

It is important to note, however, that there was a trend towards a greater quantity and quality of observations during the pre-1960s period, when a particular storm crossed the coast. This was a result of a greater concentration of population in coastal areas. As such, there was an increased likelihood of identifying and obtaining direct surface measurements for landfall events. This means that a greater level of reliability can be attached to the landfall record than for the entire region for the era prior to satellite observations. Indeed, Holland (1981) noted that most landfall events in the eastern Australian region dating back to 1910 would likely have been identified because of a dense network of stations along this coast. The analysis presented here is based on the assumption that landfall events are relatively well represented in the pre-1960s record.

Even over the post-1960 instrumental period, further improvements in observational technologies and analysis techniques have had an effect on the number of tropical cyclone events classified. Nicholls (1992) regressed first differences of seasonal tropical cyclone numbers in the entire Australian region and the SOI to reduce the effect of trends due to these changes in observational practices. Nicholls et al. (1998) attributed the presence of an apparent shift to a lower frequency of events during the mid-1980s to an increased ability to discriminate between tropical cyclones and other low-pressure systems. Buckley et al. (2003) identified a shift to a decreased period of activity in the late 1970s for the case of Tasman Sea tropical cyclones, which was also attributed to changes in observational practices. Therefore, following Nicholls et al. (1998), the analysis presented here used only tropical cyclones that attained a minimum central pressure of $\leq 990 \mathrm{hPa}$ at some stage during their lifetime. Figure 2 shows the time series of counts of these events for the period $1910 / 1911$ to $2004 / 2005$.

The seasonal ENSO index used in this analysis was obtained from a 4-month average of the SOI, the normalised mean sea-level pressure difference between Darwin and Tahiti, for the period August-November. Given that tropical cyclone events that comprise the
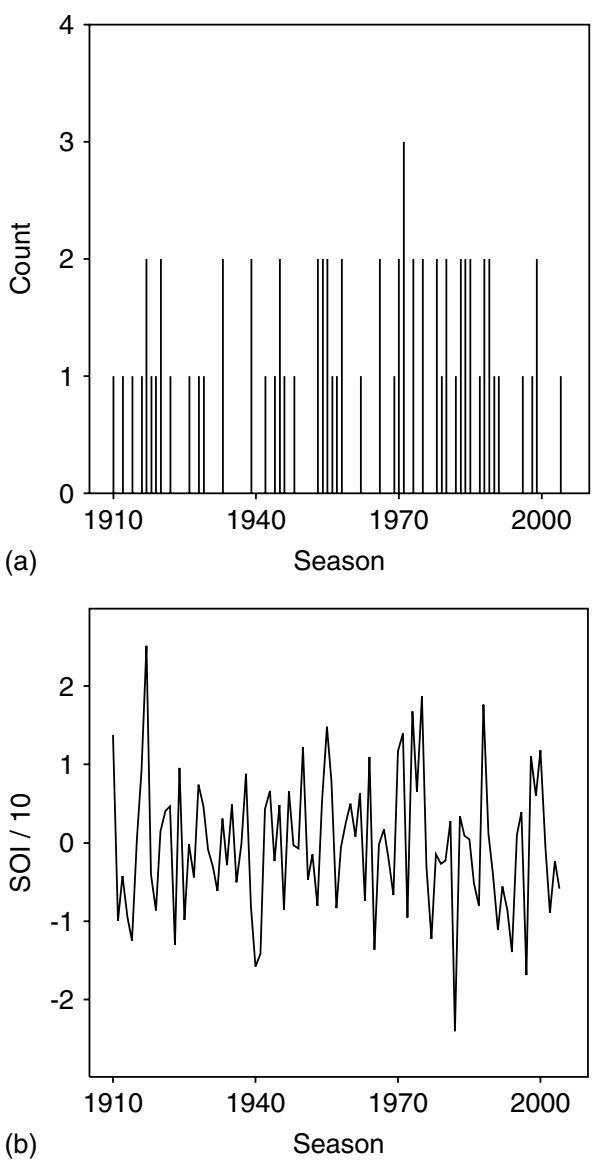

Figure 2. Time series of (a) Queensland tropical cyclone landfall numbers (with minimum central pressures of at least $990 \mathrm{hPa}$ ), and (b) averaged August-November SOI, for the period 1910/1911-2004/2005.

landfall record occurred exclusively over the months December to May, the August to November SOI is taken here to be a suitable pre-season index. Monthly values of the SOI were obtained from the BoM (http://www.bom.gov.au/climate/current/soihtm1.shtml). A 4-month mean is used in preference to a single monthly value because monthly SOI values are more likely to contain variability that is unrelated to the ENSO phenomena itself (Trenberth, 1984). This SOI time series is also shown in Figure 2.

\section{REGRESSION ANALYSIS}

The relationship between seasonal landfall counts and the SOI is investigated using a regression analysis. Nicholls et al. (1998) employed a linear regression on the August SOI and storm counts in the entire Australian region as a means to forecast seasonal activity. Both Solow and Nicholls (1990) and McDonnell and Holbrook (2004) adopted Poisson regression models in a similar context using the September SOI. Poisson regression is a special case of the generalised linear model (GLM), which is an extension of the classical linear regression to include response variables that follow any distribution in the exponential family (McCullagh and Nelder, 1989). 
A Poisson process has a single parameter $\lambda>0$, here specifying the mean rate of event occurrence per season. The Poisson regression is used to model a set of counts that are observed in seasons $i=1, \ldots, n y_{i} \in$ $0,1,2, \ldots, \infty$, with the parameter $\lambda_{i}$ estimated as a function of a vector of predictor variables $x_{i}^{\prime}$ :

$$
\begin{aligned}
& y_{i} \sim \text { Poisson }\left(\lambda_{i}\right) \\
& \ln \left(\lambda_{i}\right)=x_{i}^{\prime} \beta
\end{aligned}
$$

The natural logarithm is the canonical link function for the Poisson GLM (McCullagh and Nelder, 1989), while $\beta$ is a vector of regression parameters associated with the predictors.

The GLM is typically fit by way of a maximum likelihood procedure to estimate the parameter vector $\beta$ by maximising the likelihood function for the model $\ell(y \mid \beta)$. An alternative approach that is adopted here follows Elsner and Jagger (2004) in using a Bayesian strategy to fit the GLM. Fundamental to the Bayesian approach is that model parameters are treated not as fixed values but as random variables. These parameters are assigned a prior density $\pi(\beta)$, about which information is expressed without reference to the data. Inference concerning the parameters is then based on the posterior distribution $\pi(\beta \mid y)$, which according to Bayes' Theorem can be obtained from:

$$
\pi(\beta \mid y) \propto \pi(\beta) \ell(y \mid \beta)
$$

To implement a Bayesian approach it requires that prior distributions be specified for the model parameters. This leads to the following model structure used in this study:

$$
\begin{aligned}
& y_{i} \sim \operatorname{Poisson}\left(\lambda_{i}\right) \\
& \ln \left(\lambda_{i}\right)=\beta_{0}+\beta_{1} \text { SOI } \\
& \beta \sim \operatorname{MVN}(\phi, \Sigma)
\end{aligned}
$$

This particular model structure represents a threestage hierarchical Bayesian approach (see e.g. Wikle and Anderson, 2003). At the first stage, a Poisson process with rate parameter $\lambda_{i}$ is specified for the data describing the process. At the second stage, a Poisson regression is used to relate the log-transformed rate parameter to the SOI, with parameters $\beta=\left(\beta_{0}, \beta_{1}\right)$ describing the strength of the association. In fitting this particular model, the seasonal SOI values are divided by a factor of 10 to allow for a more convenient representation of results. Finally, at the third stage, a multi-variate normal (MVN) prior distribution having mean vector $\phi$ and covariance matrix $\Sigma$ is assigned to the model parameters. The choice of a MVN prior distribution follows that used by Elsner and Jagger (2004).

To complete the model specification requires values for $\phi$ and $\Sigma$ of the MVN prior. In the non-informative case, values for these parameters are chosen to reflect lack of prior information, leading to the specification of a vague prior distribution. In the informative case, prior information can be derived from various sources, including expert opinion, spatial information, and historical data. Owing to the relatively short length of instrumentally recorded data available for fitting the Poisson GLM, it is important to adopt an approach that maximises use of all existing information. For this reason, historical counts from the pre-1960 period are used here to obtain an informative prior.

Following Elsner and Jagger (2004), a bootstrap resampling procedure is used to estimate prior parameters from historical counts for the period 1910/1911-1959/ 1960. This is achieved by fitting a GLM of the form, $\ln \left(\lambda_{i}\right)=\beta_{0}+\beta_{1}$ SOI, individually to 1000 bootstrap samples of the historical counts. Fitting is done through maximum likelihood. The purpose of using the bootstrap re-sampling procedure is to allocate a likely range of uncertainty to estimating model parameters from historical counts. For each bootstrap sample, the parameter estimates $\beta^{*}=\left(\beta_{0}{ }^{*}, \beta_{1}{ }^{*}\right)$ are retained, allowing $\phi$ and $\Sigma$ to be calculated from the series $\beta_{1}{ }^{*}, \ldots, \beta_{1000}{ }^{*}$. Sampling distributions of the parameter estimates based on these bootstrap samples are shown in Figure 3.

Bayesian inference for the model shown in Equation (3) is relatively simple with the use of Markov chain
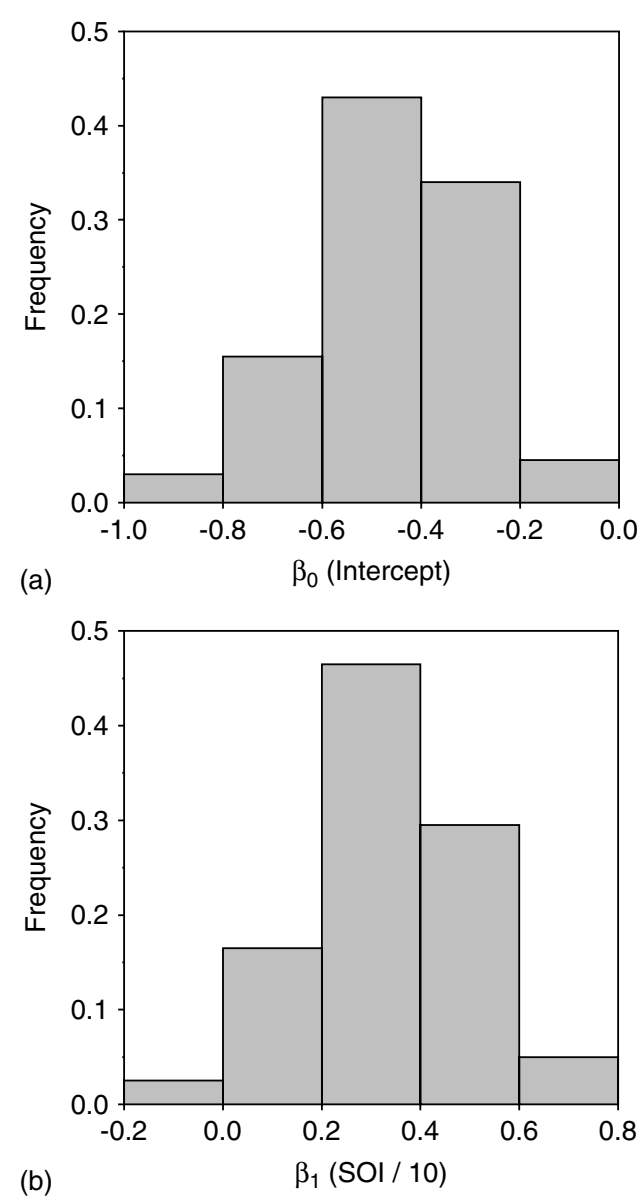

Figure 3. Sampling distributions of regression coefficients (a) intercept term, and (b) SOI term, from GLM fit to bootstrap samples of historical counts (1910/1911-1959/1960). 
Monte Carlo (MCMC) techniques. MCMC techniques offer an indirect way of obtaining an estimate of the posterior distribution. A straightforward approach is to use a Gibbs sampling algorithm (Gelfand and Smith, 1990), which attempts to simulate a Markov chain in such a way that its equilibrium distribution converges to the target posterior $\pi(\beta \mid y)$. Gibbs sampling involves an iterative procedure that generates samples from the posterior distribution by successively updating the individual parameter components conditionally on the current values of the other parameters (see e.g. Elsner and Jagger, 2004; Wikle and Anderson, 2003). The Gibbs sampling scheme used here involved a 5000 iteration burn-in period with 10000 subsequent updates used to summarise posterior distributions of the regression parameters. Convergence was verified by visual inspection of the chains for stability and by repeating the process for several different initial values.

Using this procedure, the GLM was fit to counts from the instrumental era (1960/1961-2004/2005) with the informative prior derived from historical counts using the bootstrap procedure described previously. Plots of the posterior densities for the regression parameters are shown in Figure 4. These were obtained by applying a standard Gaussian kernel density estimator to the
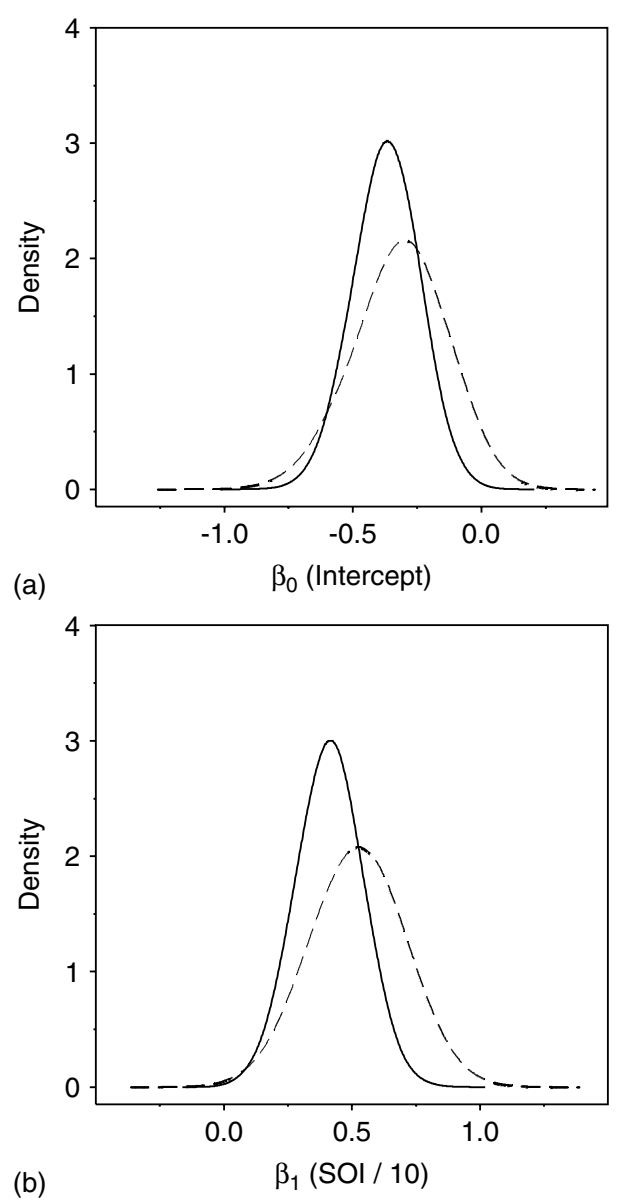

Figure 4. Posterior distributions of Bayesian GLM regression coefficients (a) intercept term, and (b) SOI term, based on non-informative (dashed line) and informative (solid line) priors. output MCMC samples. The posterior density for $\beta_{1}$, representing the SOI term, is seen to have its mass largely greater than zero, meaning the majority of MCMC output samples for this term are in the positive domain.

To check the sensitivity of the results to the prior specification, the Bayesian GLM was also fit with a noninformative prior. This places emphasis solely on the reliable sample information (i.e. instrumental record) in the estimation process. The resulting posterior densities are also plotted in Figure 4. Comparison of the informative and non-informative prior cases indicates that inclusion of historical data as prior information shifts the posterior density for $\beta_{1}$ slightly towards zero, indicating that the influence of ENSO was perhaps weaker during the historical period. The posterior mean for $\beta_{1}$ with the historical prior is 0.41 , while for the non-informative prior case it is 0.52 . It can also be seen from Figure 4 that posterior distributions are less diffuse for the informative prior case, indicating that uncertainty in parameter estimates is reduced by the inclusion of historical information.

In order to examine the model's predictive capacity, two scenarios representing extremes of ENSO are presented as examples. In the Bayesian approach, the posterior predictive density for some future observation $z$ with density function $f(z \mid \beta)$ is defined by

$$
f(z \mid y)=\int f(z \mid \beta) \pi(\beta \mid y) \mathrm{d} \beta
$$

The posterior predictive distribution accounts for uncertainty in both parameter estimates and that due to the variability of future observations. Direct calculation of Equation (4) can again be avoided by using the output posterior MCMC samples.

Figure 5 gives predictive distributions showing the probability of observing a specified number of events in a season, given SOI values of -25 and 25 . These values of the SOI correspond respectively to a major El Niño and a major La Niña event. As expected, these show a marked increase in the probability of observing one or more events during a major La Niña event than during a major El Niño event. For instance, the probability of observing two or more landfalls in a season when the $\mathrm{SOI}=25$ is about $54 \%$ higher than when the $\mathrm{SOI}=-25$.

\section{MODEL VALIDATION}

Model skill in the forecast context was tested using a leave-one-out cross-validation procedure. Cross-validation works by successively omitting an observation from the data set, then re-fitting the model with the remaining data, and using these estimates to obtain a prediction of the omitted observation (Solow and Nicholls, 1990; Elsner and Jagger, 2006). From this, the mean-squared error (MSE) can be calculated from observed and predicted values. As the observed storm counts were found to exhibit no serial correlation at lags of 1-10 seasons, 


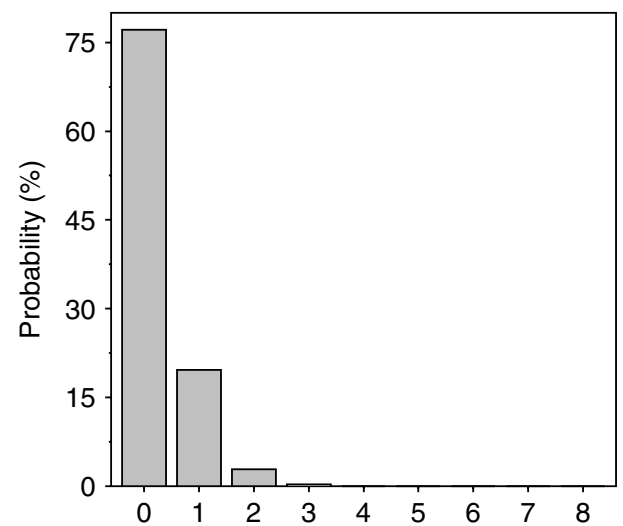

(a)

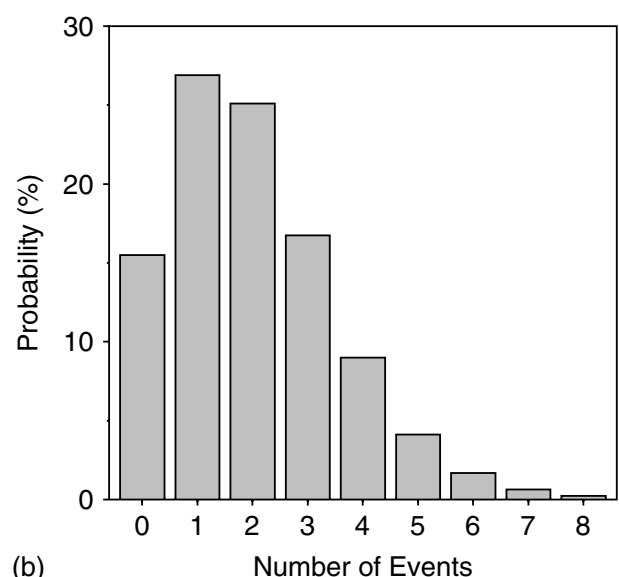

Figure 5. Predictive distributions of the probability of observing tropical cyclone landfalls under two ENSO states (a) $\mathrm{SOI}=-25$ (strong El Niño) and (b) SOI $=25$ (strong La Niña).

it can be assessed that they are independent from season to season, hence the standard approach of removing a single count at a time is used. Elsner and Jagger (2006) discuss the use of cross-validation for seasonal prediction of US hurricanes based on the application of a Bayesian GLM. A similar approach is employed here to assess three model strategies for the prediction period 1960/1961-2004/2005: the first with the informative historical prior, the second with a non-informative prior, and a third model based on climatology. The climatology model uses no ENSO predictor and is obtained by fitting a Poisson distribution to observed counts with a non-informative prior. The MCMC fitting process used to estimate parameters for each model strategy is identical to that previously described.

Table I shows the results of the cross-validation procedure. The MSE for the model using the informative prior is 1.437 , it is 1.555 for the uninformative prior, and 1.697 for climatology. Hence, it can be assessed that the model with the informative prior outperforms that using the non-informative prior as well as that based on climatology. Table I also shows MSE values for seasons in which 0,1 , or 2 counts were observed. Although the sample sizes are relatively small for these cases, again it can be seen that informative prior model obtains lower MSE values.
Table I. Summary of cross-validated mean-squared errors (MSE) of prediction of seasonal tropical cyclone activity for models based on climatology, non-informative prior, and informative historical prior.

\begin{tabular}{lcccc}
\hline \multirow{1}{*}{ Model } & \multicolumn{4}{c}{ Number of events } \\
\cline { 2 - 5 } & $\geq 0$ & 0 & 1 & 2 \\
\hline Climatology & 1.697 & 1.563 & 0.876 & 2.286 \\
Non-informative & 1.555 & 1.343 & 0.899 & 2.282 \\
Informative & 1.437 & 1.147 & 0.831 & 2.212 \\
\hline
\end{tabular}

The cross-validation exercise also showed that the informative prior model outperformed climatology in $73 \%$ of the sample seasons and the non-informative prior model in $71 \%$ of sample seasons. As expected, the climatology model worked well in seasons where the observed count is near the mean rate for the series and poorly when it is not close to this rate. On the basis of the MSE values in Table I, it appears that the informative prior model achieves most of its improved performance through prediction of seasons where there were no landfall events.

\section{TREND ANALYSIS}

The results obtained from fitting the Poisson regression with informative and non-informative priors indicated that the strength of the relationship between ENSO and the tropical cyclone activity was possibly weaker during the historical era. This raises the possibility of variation in the ENSO relationship with tropical cyclone landfalls over time. A relatively simple approach adopted here to examine this was to fit the regression to a moving window of the tropical counts.

Figure 4 shows that the mass of the posterior distribution for the $\beta_{1}$ term is greater than zero. A suitable test of significance can therefore be established by requiring a high proportion of the posterior samples generated from the MCMC scheme to be greater than zero for $\beta_{1}$ to be a significant term in the model. By applying this criterion to the GLM fit to a moving window of the record, variation in the strength of the relationship over time can be examined. This approach is similar to that applied by Elsner et al. (2001), who used maximum likelihood fitting of a Poisson GLM to a moving window of US hurricane counts with the SOI as a covariate to examine secular variability in the relationship over the twentieth century.

Figure 6 shows the application of this approach with 30- and 40-year moving windows of the record from $1910 / 1911$ to $2004 / 2005$. The $p$-values measure the significance of the SOI term in the model, calculated as the fraction of post-convergence MCMC samples greater than zero. Higher $p$-values thus imply that the inclusion of the SOI offers no improvement over a null model without the ENSO predictor (climatology). Each $p$-value in Figure 6 corresponds to a successive fit of the 


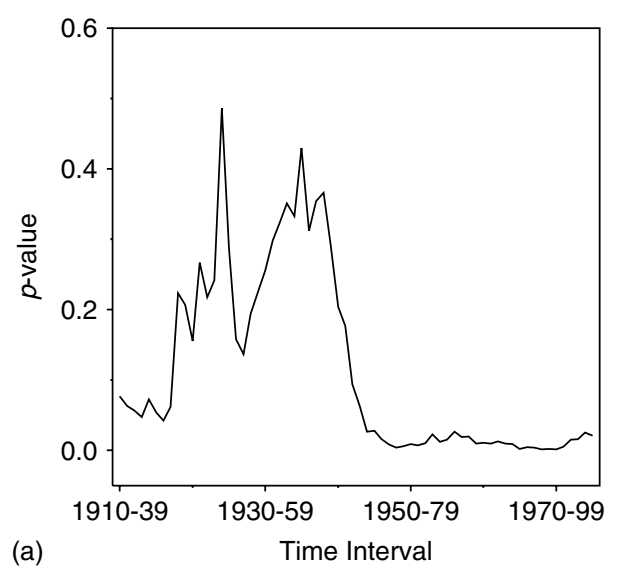

(a)

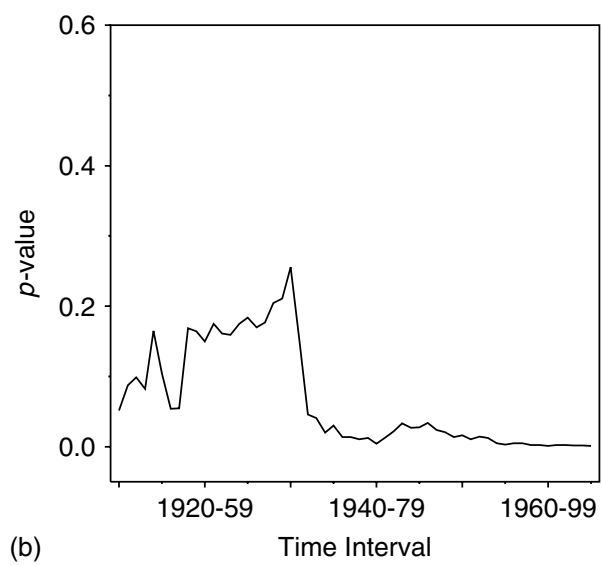

Figure 6. Significance of the relationship between tropical cyclone landfalls and ENSO as measured over (a) 30-year and (b) 40-year moving windows of the time series.

model in Equation (3) using a non-informative prior. For instance, the first point plotted in Figure 6(a) gives the $p$-value for the fit to the period 1910/1911-1939/1940, the second for the period 1911/1912-1940/1941, and so on up to 1975/1976-2004/2005. No formal level is given to assess significance; instead, the analysis is used to highlight possible periods when the relationship was weaker. Interestingly, the plots highlight a pattern whereby there is an absence of a strong relationship between ENSO and Queensland landfall activity over much of the first half of last century.

\section{SUMMARY AND DISCUSSION}

The analysis presented here demonstrates that methodologies are available to incorporate pre-1960s historical information as a means to better assess the relationship between tropical cyclone landfalls and ENSO in Queensland. In particular, the adoption of a Bayesian modelling strategy to incorporate historical counts in fitting a Poisson regression model with the SOI as a predictor is shown to have several advantages. This includes a reduction in uncertainty of parameter estimates and smaller mean-squared prediction errors. This subsequently allows predictions of seasonal activity (e.g. Figure 5) to be made with greater confidence than those derived from only the instrumental record or those based on climatology. The output of a Bayesian analysis also allows predictions to be made across the range of possible outcomes in terms of a probabilistic estimate.

Regardless of which prior specification is used though, there still remain several seasons in which an SOI-based predictor of landfall activity performs poorly. This occurs where there is a discrepancy between an observed count and the established relationship between the SOI and Australian region tropical cyclone activity. For instance, during the 1964/1965 season, no landfall event was observed despite a relatively high positive value of the mean August-November SOI of 10.95. Over the period 1978/1979-1989/1990, there were also a number of seasons where observed landfall activity was relatively high when the SOI was indicative of neutral ENSO conditions. There are several possible explanations for this, including errors in the data set of counts, the need for additional predictors, the possibility of trends in the relationship between ENSO and tropical cyclone activity, or a combination of these and other factors.

The analysis has highlighted the lack of a significant association between the SOI and landfalls in Queensland during part of the first half of the last century. A similar conclusion was reached by Grant and Walsh (2001), who correlated landfall numbers with the SOI for three separate periods; 1920-1940, 1941-1966, and 1967-1996, and found no significant correlation in the period 1920-1940. Whether this reflects an actual trend of decadal variability in the relationship between ENSO and tropical cyclone landfalls or is simply indicative of the less accurate nature of storm counts in the earlier record is difficult to ascertain. It is interesting to note though that Elsner et al. (2001) also found a period where the relationship between ENSO and US hurricane activity was weakened, which they attributed to the influence of the North Atlantic Oscillation.

Likewise, a physical reason for the trend observed here is indeed a possibility because it is well known that the strength of ENSO teleconnections with climate patterns vary on decadal timescales. In particular, Allan et al. (1996) refer to the 1920-1940 period as one characterised by conditions close to climatology, unlike the periods prior to and following this where strong ENSO-phase activity was in evidence. A significant weakening in the relationship between the SOI and northeast Australian rainfall was observed during the 1931-1945 period (Cai et al., 2001). Furthermore, Hendy et al. (2003) using luminescent banding in Porites coral cores at sites in the central great barrier reef (GBR) as a proxy for Burdekin river runoff and Queensland summer rainfall found that these variable ENSO teleconnections have been present over at least the last 400 years.

While the mechanisms behind this variability are yet to be fully explained, Power et al. (1999) recently suggested that the strength of ENSO teleconnections with certain aspects of eastern Australian climate are dependent on low-frequency Pacific SST anomalies associated 
with the Interdecadal Pacific Oscillation (IPO). In particular, Power et al. (1999) found a weakening in established relationships between ENSO and climate in IPO positive periods during which SSTs are generally lower in the southwest Pacific region. This is somewhat consistent with the trend in Figure 6, whereby the weakening of the relationship between ENSO and Queensland landfalls tends to occur during the IPO positive phase. This is most apparent for the IPO positive phase of 1924/1925-1943/1944, although less so for the IPO positive phase beginning around 1979/1980. This suggests the IPO may be a factor in modulating the strength of the ENSO relationship with Queensland tropical cyclone landfalls. If this were indeed the case, then ENSO-based predictions of seasonal landfall activity during IPO positive phases may perform poorly.

It should be noted though that because of the relatively small sample of landfalls, sampling variability likely influences the results of the trend analysis. Random fluctuations in small samples may either mask the presence of a trend, or, alternatively, result in mistakenly interpreting the presence of a trend. This combined with the uncertain nature of historical records makes trend detection difficult. Nicholls et al. (1998) also stressed on caution in interpreting the results of a trend analysis of tropical cyclone counts where there have been major changes in observational practices over time. It would therefore be imperative to investigate if the result reflects similar patterns in Australian and Coral Sea region tropical cyclone activity before a clearer picture on whether this trend is natural or artificial can be obtained.

\section{REFERENCES}

Allan R, Lindesay J, Parker D. 1996. El Niño Southern Oscillation and Climatic Variability. CSIRO Publishing: Collingwood.

Basher RE, Zheng X. 1995. Tropical cyclones in the southwest Pacific: spatial patterns and relationships to Southern Oscillation and sea surface temperature. Journal of Climate 8: 1249-1260.

Broadbridge LW, Hanstrum BN. 1998. The relationship between tropical cyclones near Western Australia and the Southern Oscillation Index. Australian Meteorological Magazine 47: 183-189.

Buckley BW, Leslie LM, Speer MS. 2003. The impact of observational technology on climate database quality: tropical cyclones in the Tasman Sea. Journal of Climate 16: 2640-2645.

Cai W, Whetton PH, Pittock AB. 2001. Fluctuations of the relationship between ENSO and northeast Australian rainfall. Climate Dynamics 17: $421-432$.
Callaghan J. 2005. Tropical Cyclone Impacts Along the Australian East Coast from November to April 1858 to 2004. Bureau of Meteorology: Brisbane.

Elsner JB, Bossak BH. 2001. Bayesian analysis of U.S. hurricane climate. Journal of Climate 14: 4341-4350.

Elsner JB, Jagger TH. 2004. A hierarchical Bayesian approach to seasonal hurricane modeling. Journal of Climate 17: 2813-2827.

Elsner JB, Jagger TH. 2006. Prediction models for annual U.S. hurricane counts. Journal of Climate 19: 2935-2952.

Elsner JB, Bossak BH, Niu X. 2001. Secular changes to the ENSOU.S. hurricane relationship. Geophysical Research Letters 28: 4123-4126.

Gelfand AE, Smith AFM. 1990. Sampling-based approaches to calculating marginal densities. Journal of the American Statistical Association 85: 398-409.

Grant AP, Walsh KJE. 2001. Interdecadal variability in north-east Australian tropical cyclone formation. Atmospheric Sciences Letters 2: $9-17$.

Hendy EJ, Gagan MK, Lough JM. 2003. Chronological control of coral records using luminescent lines and evidence for nonstationary ENSO teleconnections in northeast Australia. Holocene 13: $187-199$.

Holland GJ. 1981. On the quality of the Australian tropical cyclone data base. Australian Meteorological Magazine 29: 169-181.

McCullagh P, Nelder JA. 1989. Generalized Linear Models, 2nd edn. Chapman and Hall: London.

McDonnell KA, Holbrook NJ. 2004. A Poisson regression model approach to predicting tropical cyclogenesis in the Australian/southwest Pacific Ocean region using the SOI and saturated equivalent potential temperature gradient as predictors. Geophysical Research Letters 31: L20110, DOI: 10.1029/2004GL020843.

Nicholls N. 1979. A possible method for predicting seasonal tropical cyclone activity in the Australian region. Monthly Weather Review 107: $1221-1224$

Nicholls N. 1984. The Southern Oscillation, sea-surface-temperature, and interannual fluctuations in Australian tropical cyclone activity. Journal of Climatology 4: 661-670.

Nicholls N. 1992. Recent performance of a method for forecasting Australian seasonal tropical cyclone activity. Australian Meteorological Magazine 40: 105-110.

Nicholls N, Landsea CW, Gill J. 1998. Recent trends in Australian region tropical cyclone activity. Meteorology and Atmospheric Physics 65: 197-205.

Power S, Casey T, Folland C, Colman A, Mehta V. 1999. Interdecadal modulation of the impact of ENSO on Australia. Climate Dynamics 15: 319-324.

Revell CG, Goulter SW. 1986. South Pacific tropical cyclones and the Southern Oscillation. Monthly Weather Review 114: 1138-1145.

Solow A, Nicholls N. 1990. The relationship between the Southern Oscillation and tropical cyclone frequency in the Australian region. Journal of Climate 3: 1097-1101.

Trenberth KE. 1984. Signal versus noise in the Southern Oscillation. Monthly Weather Review 112: 326-332.

Wikle CK, Anderson CJ. 2003. Climatological analysis of tornado report counts using a hierarchical Bayesian spatiotemporal model. Journal of Geophysical Research 108D: 9005, DOI: $10.1029 / 2002 J D 002806$. 